\title{
Cooperative Sensing of Wideband Cognitive Radio: A Multiple-Hypothesis-Testing Approach
}

\author{
Sau-Hsuan Wu, Member, IEEE, Chao-Yuan Yang, and D.-H. Tina Huang
}

\begin{abstract}
A low-complexity decision fusion method is proposed for cooperative spectrum sensing (SS) of wideband cognitive radio (CR) using multiple-hypothesis testing. To maintain the quality of SS in multiple channels, performance indices of false-sensing rate and false-ignorance rate are defined for the Benjamini-Hochberg (BH) procedure to control the false-alarm ratio (FAR) and missing ratio (MR) of SS, respectively. Extended from these results, a double BH procedure is further studied in an attempt to simultaneously attain a low FAR and MR. In addition to identifying the availability of channels, a signal strength-estimation scheme that features multiple-hypothesis testing is also proposed to classify the level of the SNR for cooperative SS. Simulation results show that the double BH procedure can suppress both the FAR and the MR at a high SNR while maintaining a considerable FAR in the low-SNR regime. As such, the system can enjoy throughput enhancement by allowing more active cognitive access at a high SNR and still prevent introducing excessive multiple-access interference to the primary users when their SNRs are weak. On the other hand, for SNR classification, simulations also show that the SNR can be classified within one level, plus or minus the true strength with more than a $96 \%$ chance. This allows the system to employ more generic protocols such as concurrent transmissions for cognitive access.
\end{abstract}

Index Terms-Benjamini-Hochberg procedure, cooperative spectrum sensing, multiple hypothesis testing, wideband cognitive radio.

\section{INTRODUCTION}

$\mathbf{I}$ $\mathrm{N}$ THE PAST decade, cognitive radio (CR) has emerged as an important concept in wireless communications. CR-related techniques have found applications in dynamic spectrum access (DSA) and coexistence, radio resource and spectrum management, and interoperability in infrastructureless wireless networks. Wireless communication standards such as WiFi (IEEE 802.11), Zigbee (IEEE 802.15.4), and WiMAX (IEEE 802.16) have also incorporated some simple CR properties in their options. In addition, IEEE 802.22 is calling for contributions to define the first international standard for CR [1].

Manuscript received May 22, 2009; revised September 6, 2009. First published February 25, 2010; current version published May 14, 2010. This work was supported by the National Science Council, Taiwan, under Grant NSC 962219-E-009-017. This paper was presented in part at the IEEE Asia Pacific Wireless Communication Symposium, Sendai, Japan, August 2008. The review of this paper was coordinated by Prof. H. Zhang.

The authors are with the Department of Communication Engineering, National Chiao Tung University, Hsinchu 300, Taiwan (e-mail: sauhsuan@ cm.nctu.edu.tw; yuanppp.cm95g@nctu.edu.tw; dinhwa.cm94g@nctu.edu.tw).

Color versions of one or more of the figures in this paper are available online at http://ieeexplore.ieee.org.

Digital Object Identifier 10.1109/TVT.2010.2043967
One of the enabling technologies for CR is DSA, through which the unoccupied channels in the licensed spectrum can be exploited for the unlicensed access of secondary users. To protect the licensed users (who are also referred to as the primary users) from large multiple-access interference (MAI) in CR systems, cognitive users (secondary users) may collaboratively identify the availability of channels to improve the reliability of spectrum sensing (SS) [2]. Based on this idea, a clusterbased sensing method is proposed in [3], where the cluster head with the best channel condition to the fusion center reports the final decision of its cluster to the fusion center. In addition, the average number of reporting bits is further studied in [4] when considering a nondecision region for the energy detector.

The aforementioned works basically study cooperative SS for a single narrow-band channel and take the logic OR of the decision bits from different observers to form the final decision at the fusion center. In contrast, cooperative SS is relatively less studied from a system perspective for wideband multichannel CR systems. Based on multiple-hypothesis testing, a simple decision rule is proposed in [5] for the cooperative SS of multiple channels, making use of the Benjamini-Hochberg $(\mathrm{BH})$ procedure [6] on the energy feedback (EF) of cooperative observers. As opposed to the conventional SS approaches that work on a single subband only, [5] takes the individual decisions from all observers of all subbands to jointly decide the availability of subbands, hence providing control on the decision errors from the system's viewpoint.

A common issue in multiple-hypothesis testing is the control of type-I errors. Bonferroni-type procedures [7]-[11] typically control the familywise error rate (FWE), i.e., the probability of erroneously rejecting any of the true null hypotheses, which often leads to conservative results when the number of hypotheses increases. To resolve this problem, Benjamini and Hochberg [6] propose a new type of procedure to control the false-discovery rate (FDR) out of the declared nontrue null hypotheses. As the FDR is close to the error rate, controlling the FDR also provides control on the error rate to a certain degree. Furthermore, the proposed $\mathrm{BH}$ procedure does guarantee holding the FDR under a predefined significance level when used on continuous test statistics. Due to the effectiveness of the BH procedure, it is further extended to test statistics with different characteristics, e.g., statistics with positive regression dependence [12]. Furthermore, for discrete test statistics, it is also proven in [12] that the FDR can be controlled at a level even lower than that using continuous test statistics. Motivated by the performance and cost effectiveness of decision feedback (DF), we extend the result in [5] and investigate herein, from a broader perspective, cooperative SS for wideband CR systems. 
The objective of this paper is twofold: 1) to seek an appropriate application of the $\mathrm{BH}$ procedure such that a balanced tradeoff between the false-alarm ratio (FAR) and the missing ratio (MR) can be achieved in cooperative SS for wideband CR systems and 2) to have a more precise classification of the SNR than yes or no such that cognitive access can be made possible in a more generic manner, such as concurrent transmissions of the secondary users' signals when the SNRs of the primary users allow them [13], [14]. To meet the first objective, we employ the $\mathrm{BH}$ procedure to control the performance indices of false-sensing rate (FSR) and false-ignorance rate (FIR) derived from the notion of FDR in an attempt to suppress the FAR and MR, respectively. By controlling the FSR, the FAR of SS can be suppressed as well, which, in principle, will result in more active cognitive access. On the other hand, controlling the FIR also helps suppress the MR of SS, leading to lower MAI to the primary users. Based on the $\mathrm{BH}$ procedures for controlling the FSR and FIR, a mixture of the two procedures, which is referred to as the double $\mathrm{BH}$ procedure, is also proposed to seek for a proper balance between the FAR and MR of cooperative SS.

To classify the SNR, however, the SNR strength is first partitioned into several levels. According to these predefined SNR levels, hypotheses are then defined for each subband of the wideband CR system. With these hypotheses, two types of multiple-testing approaches are studied to decide the SNR level for each subband based on the $\mathrm{BH}$ procedure. Both the decision fusion and energy fusion methods are investigated in this regard.

To verify the performance of the proposed SS schemes in practical communication environments, three types of channel settings are considered in the numerical studies. Simulation results show that, in controlling the FSR, the proposed decision fusion method outperforms the energy fusion method in FAR, while maintaining an MR comparable to the energy fusion one. On the other hand, a low MR can be achieved by controlling the FIR when ideal SNRs of subbands are available to the fusion center. When the SNR is unavailable a priori, however, the MR can still be controlled under an acceptable level, despite losing some performance in the low-SNR regime. Furthermore, the double BH method demonstrates the lowest FAR and MR at a high SNR, while still enjoying a low MR and a relatively high FAR in the low-SNR regime. This can potentially improve the system throughput by allowing for more aggressive cognitive access in the high-SNR regime, while preventing MAI that is too excessive to the primary users in the low-SNR regime.

On the other hand, for SNR classification, simulations also show that the SNR can be classified within one level plus or minus the true SNR strength with more than a $96 \%$ chance. This allows the system to employ more generic protocols such as the concurrent transmissions of the primary and secondary users' signals for cognitive access [13], [14].

The rest of this paper is organized as follows. In Section II, we describe the system model for wideband CR systems and review the notion of FDR and the $\mathrm{BH}$ procedure for multiple-hypothesis testing. The performance indices of FSR and FIR and the corresponding BH procedures are introduced in Section III to control the FAR and MR of cooperative SS.

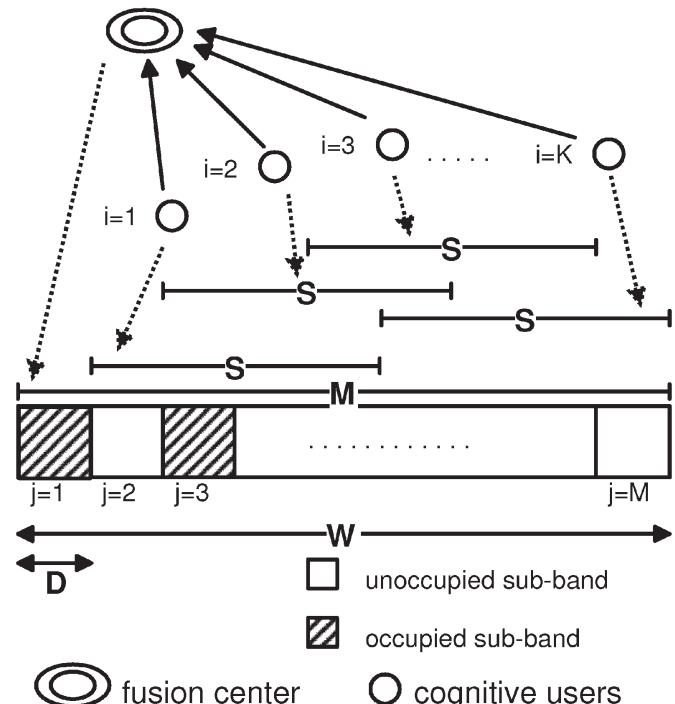

Fig. 1. System model for cooperative spectrum sensing in wideband CR systems.

Based on these results, a double $\mathrm{BH}$ procedure is proposed in Section IV in an attempt to balance the FAR and MR in cooperative SS. Furthermore, two testing approaches for SNR classification are studied in Section $\mathrm{V}$ to enable cognitive access in a more generic sense. Simulation results are presented in Section VI, followed by the conclusions in Section VII.

\section{SySTEM MOdeL}

We consider a CR system that does not officially operate over a licensed wireless band. Instead, it coexists with other radio systems that may or may not possess officially licensed frequency bands. The CR system intends to use the frequencies when they are not preoccupied by the licensed users. To access the unoccupied frequency bands, the system is equipped with a base station or a data fusion center to monitor the availability of the wireless channels. We assume that the CR system can monitor and operate over a wide frequency bandwidth of $W$ $\mathrm{Hz}$, and the overall bandwidth is divided by the system into $M$ subbands for cognitive access. As a result, each subband has a bandwidth of $D=W / M \mathrm{~Hz}$. An illustration for the CR system is shown in Fig. 1.

Now, we consider a cooperative SS scheme for the CR system. We assume that there are $K$ cognitive users in the system. Each has the same capability to scan the $M$ subbands over the operating bandwidth and randomly chooses $S$ consecutive subbands out of $M$ to observe. To decide whether the $M$ subbands are occupied or not, the fusion center first asks each user to make their own decision and report the availability for each subband the user observes. Based on the reports of all users, the fusion center makes the final decision and forms the channel occupancy vector $\bar{O} \in\{0,1\}^{M}$ for the overall $M$ subbands. The $j$ th entry of $\bar{O}$ is set as 1 if the $j$ th subband is occupied by the primary user; otherwise, it is 0 .

For each subband $j$ scanned by a secondary user $i$, there are two possible hypotheses. One assumes that the channel is occupied, which is denoted by $H_{1}$; the other assumes 
that it is unoccupied, which is denoted by $H_{0}$. Specifically, we have

$$
\begin{array}{ll}
H_{0}: & Y_{i, j}=N_{i, j} \\
H_{1}: & Y_{i, j}=X_{i, j}+N_{i, j}
\end{array}
$$

where $N_{i, j} \sim C N(0,1)$, and $X_{i, j} \sim C N(0, q)$, with $q>1$. The user $i$ reports the availability of the subband $j$ by using a simple threshold detector with the threshold set at $\lambda$. If $Y_{i, j}^{2} \geq$ $\lambda$, the user reports 1 . Otherwise, it reports 0 .

Based on the decisions bits reported by all users, the fusion center will reconstruct the channel occupancy vector $\bar{O}$. To handle the multiple-testing problem involved in forming $\bar{O}$, a traditional approach is to control the FWE. However, controlling the FWE usually comes to give a result that is too conservative when the number of hypotheses increases. To avoid this problem, we adopt the $\mathrm{BH}$ procedure to control the FDR [6]. The definition of FDR and the essence of the BH procedure are summarized below.

\section{A. FDR}

The FDR is defined as the expected proportion of the erroneously rejected true null hypotheses among the total hypotheses that are rejected. Specifically, suppose that there are $M$ hypotheses to be tested in an experiment. By some detection procedures, $N_{1}$ of $M$ hypotheses are rejected, with $F$ of them being false and $T$ of them being true decisions. The FDR is defined as

$$
\mathrm{FDR}=E[F /(F+T)]=E\left[F / N_{1}\right] .
$$

Benjamini and Hochberg show that controlling the FDR also controls the FWE in the weak sense and that the FDR is a more appropriate error rate to control than the conventional FWE in many multiple-testing problems [6].

\section{B. BH Procedure}

The BH procedure is a decision rule to control the FDR of hypothesis testing under a given significance level $\alpha$ [6]. Assume that there are $m$ hypotheses $H_{1}, H_{2}, \ldots, H_{m}$ set for the problem under investigation. Given the $p$-values $p_{1}, p_{2}, \ldots, p_{m}$, which correspond to the hypotheses $H_{1}, H_{2}, \ldots, H_{m}$, respectively, the $\mathrm{BH}$ procedure for verifying the correctness of the hypotheses is given as follows.

1) Sort the $p$-values in ascending order, and denote the ordered $p$-values as $p_{(1)} \leq p_{(2)} \leq \cdots \leq p_{(m)}$.

2) Find the largest index $i_{\max }$ such that $p_{\left(i_{\max }\right)} \leq$ $\left(i_{\max } / m\right) \alpha, 1 \leq i_{\max } \leq m$.

3) Reject all $H_{(i)}, i=1,2, \ldots, i_{\max }$.

For independent test statistics, if the $\mathrm{BH}$ procedure is conducted at a significance level $\alpha,[12]$ proves that the FDR can be controlled exactly at the level $\left(m_{0} / m\right) \alpha$ for continuous test statistics and at a level less than or equal to $\left(m_{0} / m\right) \alpha$ for discrete test statistics, where $m_{0}$ is the number of true null hypotheses among the $m$ hypotheses.

\section{Controlling the False-Sensing Rate And the FALSE-IGNORANCE RATE}

Based on the $\mathrm{BH}$ procedure, we attempt to develop schemes to control the FAR and the MR of SS in wideband CR systems. To this end, we define two controlled error rates for the $\mathrm{BH}$ procedure following the notion of FDR. One is referred to as the FSR, which serves as an alternative metric for FAR in the BH procedure. The other is the FIR, which serves as an alternative for the MR in the BH procedure. The details about the SS schemes are specified in the sequel.

\section{A. FSR}

The FSR is defined as the expected proportion of the number of falsely sensed channels over the total number of channels that are declared occupied. In CR systems, the secondary users are allowed to access a frequency band when there is no primary user using the channel. By controlling the FSR below a certain low level, a channel is less likely to be mistaken as occupied, thus allowing more access from the secondary users. This may improve the overall system throughput at the risk of introducing higher MAI to the primary users.

For controlling the FSR, the true and nontrue null hypotheses are given by (1) and (2), respectively. Under the true null hypothesis $H_{0}$, the energy $\left|Y_{i, j}\right|^{2}$ is independent of the signal strength $q$ and is exponentially distributed. Given the threshold $\lambda$, the probability for a cognitive user $i$ to report 1 (false alarm under $H_{0}$ ) to the fusion center is

$$
P_{F}=\operatorname{Pr}\left\{\left|Y_{i, j}\right|^{2} \geq \lambda \mid H_{0}\right\}=\int_{\lambda}^{\infty} e^{-y} d y=e^{-\lambda} .
$$

Now, suppose that there are $N_{j}$ observers for the subband $j \in$ $\{1, M\}$, and each reports to the fusion center about its decision on the availability of the subband. With the $N_{j}$ decision bits and $P_{F}$, we define the $p$-value $p_{j}$ for the hypothesis $H_{0}$ as the complementary cumulative distribution function (CCDF) of the binomial distribution, which is given by

$$
p_{j}=\sum_{x=x_{j}}^{N_{j}}\left(\begin{array}{c}
N_{j} \\
x
\end{array}\right)\left(e^{-\lambda}\right)^{x}\left(1-e^{-\lambda}\right)^{N_{j}-x}
$$

where $x_{j}$ is the number of $1 \mathrm{~s}$ in $N_{j}$. Based on the $p$-value, the $\mathrm{BH}$ procedure can be applied at a level $\alpha$ to decide the availability of each channel. The steps are listed below.

1) Calculate the $p$-value $p_{j}, \forall j \in\{1, M\}$, with (5).

2) Sort $p_{j}, \forall j \in\{1, M\}$, in ascending order.

3) Find the largest index $i_{\max }$ such that $p_{\left(i_{\max }\right)} \leq$ $\left(i_{\max } / M\right) \alpha$.

4) Declare subband $(j)$ occupied for the reordered indices $1 \leq(j) \leq i_{\max }$.

The performance of controlling the FSR is presented in Figs. 2(a) and 3. For comparison, the energy fusion method [5] that makes use of $\sum_{i=1}^{N_{j}}\left|Y_{i, j}\right|^{2}$ to evaluate the $p_{j}$ is also shown in the figure. Fig. 2(a) shows that both the energy fusion and decision fusion methods can control the FSR under the desired level $\alpha=0.05$, while the proposed decision fusion method can 


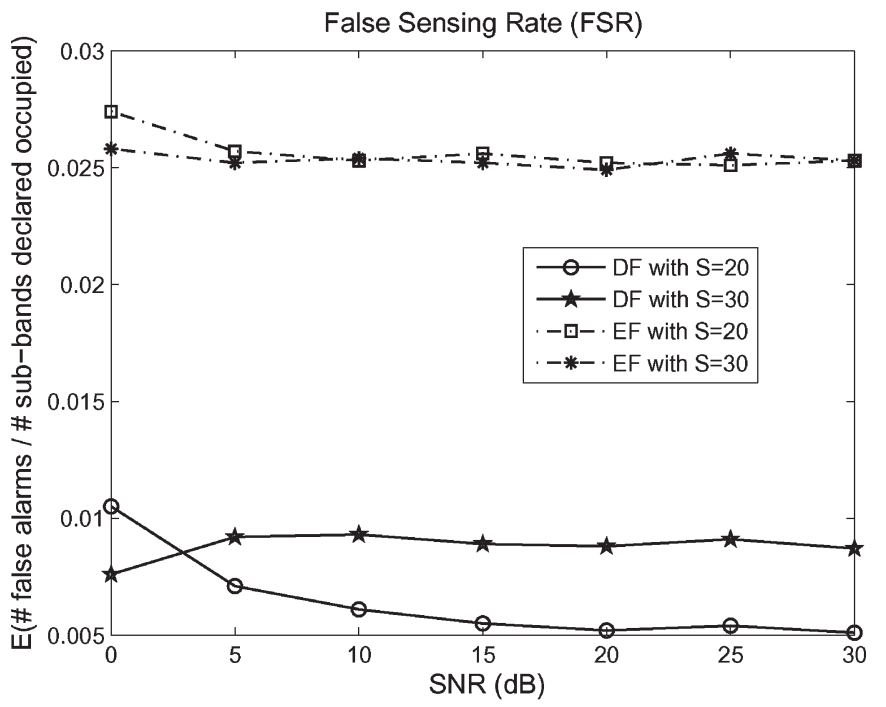

(a)

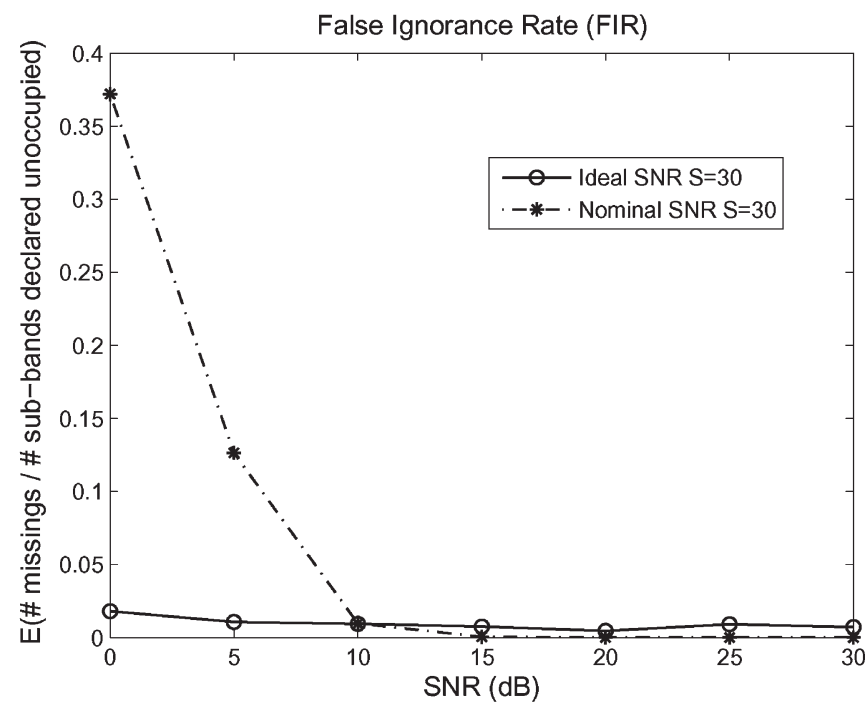

(b)

Fig. 2. FSR and FIR with $M=100, K=20, \lambda_{\mathrm{FSR}}=4, \lambda_{\mathrm{FIR}}=1$, and $\alpha=0.05$. The nominal SNR for the FIR is set at $q^{*}=10 \mathrm{~dB}$.

achieve a much lower FSR level than the energy fusion one. This advantage results from the use of discrete test statistics in the $\mathrm{BH}$ procedure, which has been proven in [12].

Fig. 3(a) shows that controlling the FSR does lead to a low FAR. However, the side effect is that the MR is high in the low-SNR regime, as shown in Fig. 3(b). This is due to the fact that the $\mathrm{BH}$ procedure tends to underestimate the signal strength to control the proportion of false alarms, causing a higher MR when the signal is weak. Opposite to the FAR, the energy fusion method outperforms the decision fusion method in MR, and more observers seem to give better results.

\section{B. FIR}

Although a low FSR may benefit the overall system throughput, Fig. 3(b) also indicates that it causes a high MR at a low SNR. As a result, the primary users will suffer from larger MAI in the low-SNR regime. To avoid this situation, suppressing the MR seems to be an option when it comes to maintaining

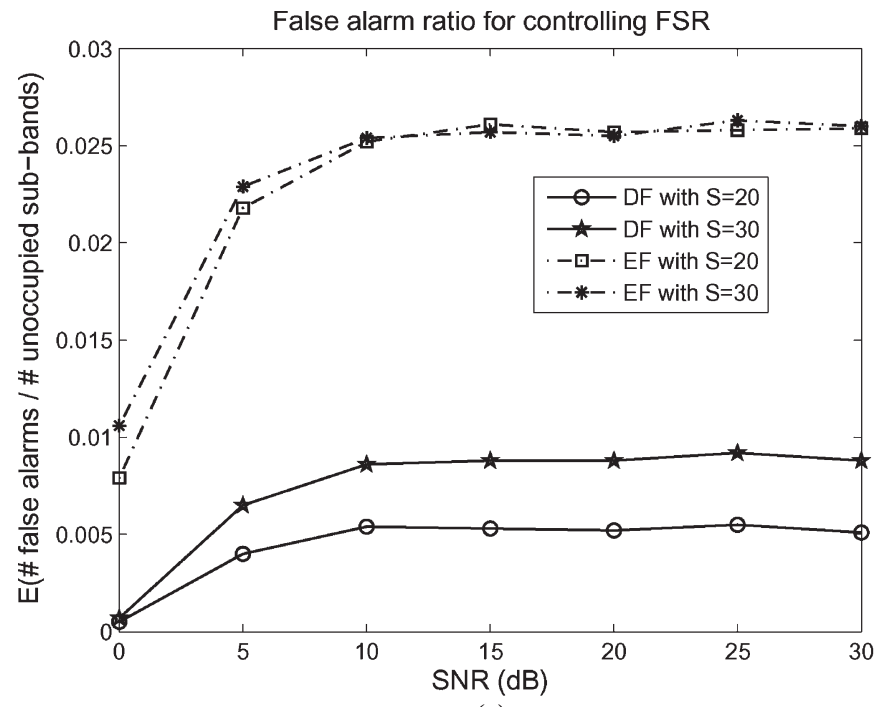

(a)

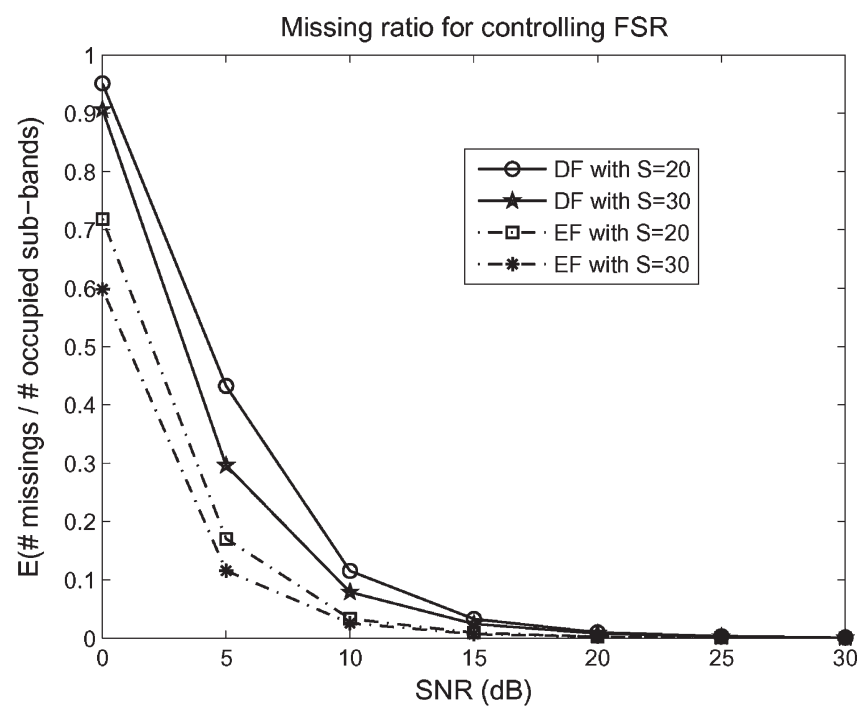

(b)

Fig. 3. FARs versus MRs of controlling the FSR with $M=100, K=20$, $\lambda_{\mathrm{FSR}}=4$, and $\alpha=0.05$

the primary users' transmissions under a tolerable interference level. To this end, we define the FIR for the $\mathrm{BH}$ procedure, which is the expected proportion of the number of falsely ignored channels over the total number of channels that are declared unoccupied.

Now, for the $\mathrm{BH}$ procedure to control the FIR, the corresponding true and nontrue null hypotheses for subband $j$ become

$$
\begin{array}{ll}
H_{0}: & Y_{i, j}=X_{i, j}+N_{i, j} \\
H_{1}: & Y_{i, j}=N_{i, j}
\end{array}
$$

where $H_{0}$ assumes that the subband is occupied, and $H_{1}$ assumes that the subband is unoccupied. Unlike the FSR, the observed energy $\left|Y_{i, j}\right|^{2}$ now has a distribution that depends on the received SNR of the user, under the true null hypothesis $H_{0}$.

Assume that $N_{i, j} \sim C N(0,1)$ and $X_{i, j} \sim C N(0, q)$, with $q>1$. Conditioned on $H_{0},\left|Y_{i, j}\right|^{2}$ is exponentially distributed with a rate parameter equal to $q+1$, which is denoted by $\left|Y_{i, j}\right|^{2} \sim \operatorname{Exp}(1 / q+1)$. As a result, the probability for a 
cognitive user $i$ to report 0 (missing under $H_{0}$ ) against the threshold $\lambda$ is

$$
\begin{aligned}
P_{M} & =\operatorname{Pr}\left\{\left|Y_{i, j}\right|^{2} \leq \lambda \mid H_{0}, q\right\} \\
& =\int_{0}^{\lambda} \frac{1}{q+1} e^{-\frac{y}{q+1}} d y=1-e^{-\frac{\lambda}{q+1}} .
\end{aligned}
$$

Given the $N_{j}$ decision bits and $P_{M}$ for subband $j$, we define the $p$-value $p_{j}$ for the hypothesis $H_{0}$ of the FIR as the CCDF of the following binomial distribution:

$$
\begin{aligned}
p_{j} & =\sum_{x=x_{j}}^{N_{j}}\left(\begin{array}{c}
N_{j} \\
x
\end{array}\right)\left(P_{M}\right)^{x}\left(1-P_{M}\right)^{N_{j}-x} \\
& =\sum_{x=x_{j}}^{N_{j}}\left(\begin{array}{c}
N_{j} \\
x
\end{array}\right)\left(1-e^{-\frac{\lambda}{q+1}}\right)^{x}\left(e^{-\frac{\lambda}{q+1}}\right)^{N_{j}-x}
\end{aligned}
$$

where $x_{j}$ is the number of 0 s out of $N_{j}$. Based on this $p$-value, the $\mathrm{BH}$ procedure can now be applied to control the FIR below a level of $\alpha$. The procedure is stated below.

1) Calculate each $p$-value $p_{j}, j \in\{1, M\}$, according to (6).

2) Sort $p_{j}, \forall j \in\{1, M\}$, in ascending order.

3) Find the largest index $i_{\max }$ such that $p_{\left(i_{\max }\right)} \leq$ $\left(i_{\max } / M\right) \alpha$.

4) Declare subband $(j)$ unoccupied for the reordered indices $1 \leq(j) \leq i_{\max }$

1) FIR Under a Nominal SNR Assumption: To evaluate the $p$-values for the FIR, the received SNR $q$ for each cognitive user of each subband must be given a priori. In practice, the SNRs are not available at the fusion center. To overcome this difficulty, a nominal SNR $q^{*}$ can be set for the fusion center to evaluate the $p$-values (7). As a result, the probability $P_{M}^{*}$ with respect to $q^{*}$ becomes a constant at the fusion center. The corresponding nominal $p$-values in (7) only depends on the $N_{j}$ of each subband and is denoted by $p_{j}^{*}, \forall j \in\{1, M\}$. Except for $P_{M}^{*}$, the rest of the $\mathrm{BH}$ procedure for controlling the FIR is exactly the same as if an ideal SNR is available at the fusion center.

Simulation results for applying the $\mathrm{BH}$ procedure to control the FIR are provided in Figs. 2(b) and 4. As shown in the figures, the FIR is held below the desired level $\alpha=0.05$ given the ideal SNR, and the MR almost diminishes to zero in Fig. 4(b). Moreover, it also makes the FAR high at a low SNR, which is similar to what the $\mathrm{BH}$ procedure for the FSR does to the MR in Fig. 3(b).

On the other hand, the simulation results for the BH procedure operating at a nominal SNR of $10 \mathrm{~dB}$ show that the MR increases when the SNR is lower than the nominal value of $10 \mathrm{~dB}$, and in the meantime, the FIR is also out of control in that region. In addition, the FAR remains at a stable value around 0.2 in Fig. 4(a). The reason why the $\mathrm{BH}$ procedure operating at a nominal SNR behaves in Figs. 2(b) and 4 is discussed below.

When $0 \leq \mathrm{SNR} \leq 10 \mathrm{~dB}$, the fusion center uses a $P_{M}^{*}$ that is smaller than the real $P_{M}$ to evaluate the $p$-values of each subband. As a result, a smaller $p$-value is obtained in comparison with the ideal SNR mode, which implies a lower availability of the subband. The MR then increases after applying the $\mathrm{BH}$

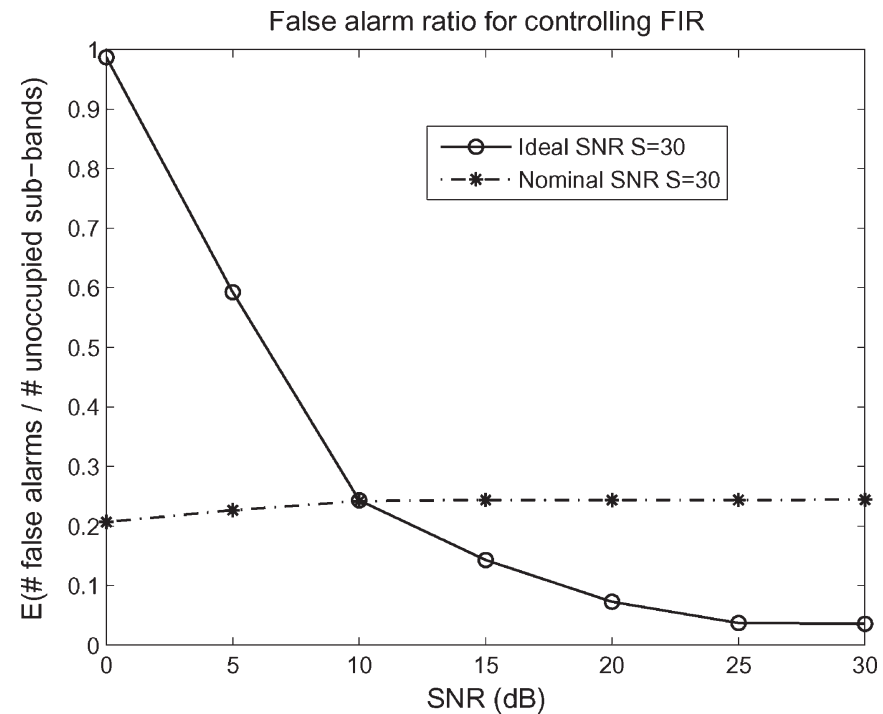

(a)

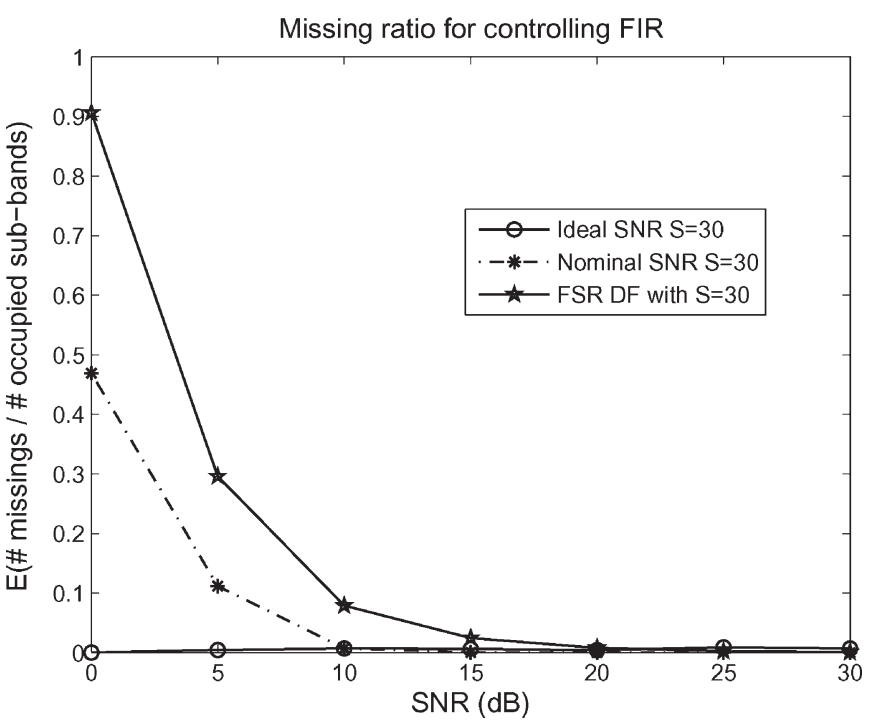

(b)

Fig. 4. FARs versus MRs of controlling the FIR with $M=100, K=20$, $\lambda_{F I R}=1$, and $\alpha=0.05$. The nominal SNR for the FIR is set at $q^{*}=10 \mathrm{~dB}$, as opposed to the cases with an ideal SNR.

procedure. For SNR $\geq 10 \mathrm{~dB}$, since $P_{M}^{*}$ is always higher than the real $P_{M}$, the MR will only be better than the ideal SNR mode. It is noted that $P_{M}^{*}$ is fixed once we set the nominal SNR at the fusion center. To compensate the effect caused by $P_{M}^{*}$, the energy threshold $\lambda$ should properly be adjusted to keep the MR in an acceptable level.

\section{Energy Thresholds for Controlling the FSR and the FIR}

As we have seen from the previous sections, the $p$-values in (5) and (7) also depend on the energy threshold $\lambda$, which, in turn, will affect the outcomes of the $\mathrm{BH}$ procedure. As a matter of fact, even the value of $x_{j}$ depends on $\lambda$. Intuitively, there will be a region for $\lambda$ such that the $\mathrm{BH}$ procedure can properly be conducted according to a predefined significance level $\alpha$. More specifically, if the smallest $p$-value is greater than $\alpha$, then all the $p$-values are greater than $\alpha$ as well. The $\mathrm{BH}$ procedure then becomes ineffective. On the other hand, if the largest 
$p$-value is smaller than $\alpha / M$, it will also lead to a failure of the $\mathrm{BH}$ procedure. However, this region for $\lambda$ is still very loose. A tighter region for $\lambda$ can be obtained if we take the single user's sensing quality into account.

Since the subbands are randomly chosen by the cognitive users, it may happen that a subband has only one observer. Under this circumstance, one should ensure that the $p$-value of that subband is still workable for the $\mathrm{BH}$ procedure. For controlling the FSR, the $p$-value of the single-observer channel has to be less than or equal to the maximum level $\alpha$ to avoid false alarms, hence leading to the lower bound of $\lambda$. On the other hand, the upper bound of $\lambda$ is obtained by having the $p$ value of the single-observer channel to be greater than or equal to the minimum level $\alpha / M$ to prevent excess MRs. According to this guideline, $\lambda$ can be chosen from $(\alpha / M) \leq e^{-\lambda} \leq \alpha$ to control the FSR, namely

$$
-\ln (\alpha) \leq \lambda \leq-\ln \left(\frac{\alpha}{M}\right) .
$$

To control the FIR, however, the $p$-value (7) of the singleobserver channel should be greater than $\alpha / M$ to suppress the MR, i.e.,

$$
\lambda^{*} \geq-(q+1) \ln \left(1-\frac{\alpha}{M}\right) .
$$

However, if one wants to implement a more conservative rule that a single-observer subband is classified as occupied if its only observer reports " 1 " to the fusion channel, then we may have a higher lower bound for $\lambda$, i.e.,

$$
\lambda^{*} \geq-(q+1) \ln (1-\alpha)
$$

\section{Double Benjamini-Hochberg Procedure For SPECTRUM SENSING}

Results from Section III show that controlling the FSR can effectively suppress the FAR of SS, while making the MR high in the low-SNR regime. On the other hand, controlling the FIR does result in a low FIR and, hence, a low MR, given the ideal SNR. However, the FAR becomes higher as well at a low SNR. These phenomena essentially result from the fact that the $\mathrm{BH}$ procedure only ensures to control the predefined error measure under the intended significance level $\alpha$; it does not control what is not defined. If the outcomes of both the $\mathrm{BH}$ procedure for the FSR and that for the FIR can jointly be considered in SS, a better compromise between the FAR and the MR may be obtained if we are able to take the advantages of both schemes.

\section{A. Double BH Procedure}

To collect outcomes from both $\mathrm{BH}$ procedures, we first change the single-threshold energy detector into a doublethreshold detector for decision fusion. The higher threshold is set for controlling the FSR, and the lower one is for controlling the FIR. When the observed energy of one subband exceeds the higher threshold, the cognitive user reports 2 for the corresponding subband. When the observed energy is below the lower threshold, it then reports 0 . Otherwise, it reports 1 when the observed energy lies in between the two thresholds.
TABLE I

Detection Rules for the Double BH Procedure

\begin{tabular}{|c|c|c|c|c|c|}
\hline \multicolumn{2}{|c|}{ Occupancy } & \multicolumn{4}{|c|}{ Decision Rules } \\
\hline$\overline{O_{F S R}}$ & $\overline{O_{F I R}}$ & FSR & FIR & OR & AND \\
0 & 0 & 0 & 0 & 0 & 0 \\
1 & 1 & 1 & 1 & 1 & 1 \\
0 & 1 & 0 & 1 & 1 & 0 \\
1 & 0 & 1 & 0 & 1 & 0 \\
\hline
\end{tabular}

At the fusion center, the $\mathrm{BH}$ procedure is tested twice to form the two occupancy vectors, i.e., $\overline{O_{\mathrm{FSR}}}$ and $\overline{O_{\mathrm{FIR}}}$, respectively. The first test of the $\mathrm{BH}$ procedure is conducted for controlling the FSR. Equations (4) and (5) are used to calculate the $p$-values of each subband. The second test of the $\mathrm{BH}$ procedure is to control the FIR according to (6) and (7) on a nominal SNR $q^{*}$. By comparing $\overline{O_{\mathrm{FSR}}}$ with $\overline{O_{\mathrm{FIR}}}$, the final decisions for the availability of subbands can be made according to the detection rules listed in Table I.

To protect the primary user from large MAI, a straightforward method is to claim the occupancy of a subband whenever $\overline{O_{\mathrm{FSR}}}$ or $\overline{O_{\mathrm{FIR}}}$ is asserted, i.e., using the OR rule. Although the MR can be suppressed in the low-SNR regime, as expected in Fig. 5(b), the side effect of the OR rule is that the FAR significantly increases, as shown in Fig. 5(a). Notice from Figs. 3(b) and 4(b) that the MRs are actually low enough in the high-SNR regime. There is no need to further suppress the MR in this SNR region with the OR rule. A proper way for decision making is to use the AND rule at a high SNR such that the FAR can properly be suppressed to enhance the system throughput. This is feasible only if the fusion center knows when to switch the decision rule at a proper SNR. Based on the $\mathrm{BH}$ procedure, an SNR-testing method is proposed in the next section to enable the rule switching, making use of the DF from the observers.

\section{B. SNR-Switching Rule}

Denote the threshold SNR to be tested by $\mathrm{SNR}_{T}$ (in decibels). To apply the $\mathrm{BH}$ procedure for hypothesis testing, the null hypothesis and the corresponding probability $P_{T}$ against the hypothesis are defined as

$H_{T}$ : The channel is occupied with a signal strength of at least $\mathrm{SNR}_{T} \mathrm{~dB}$

$$
\begin{aligned}
& P_{T}=\int_{0}^{\lambda_{T}} \frac{1}{q_{T}+1} e^{-\frac{y}{q_{T}+1}} d y=1-e^{-\frac{\lambda_{T}}{q_{T}+1}} \\
& \text { with } q_{T}=10^{\frac{\mathrm{SNR}_{T}}{10}}
\end{aligned}
$$

where the energy threshold $\lambda_{T}$ can be set according to the guidelines in Section III-C. Now, calculate the $p$-values according to

$$
\begin{aligned}
p_{j} & =\sum_{x=x_{j}}^{N_{j}}\left(\begin{array}{c}
N_{j} \\
x
\end{array}\right)\left(P_{T}\right)^{x}\left(1-P_{T}\right)^{N_{j}-x} \\
& =\sum_{x=x_{j}}^{N_{j}}\left(\begin{array}{c}
N_{j} \\
x
\end{array}\right)\left(1-e^{-\frac{\lambda}{q_{T}+1}}\right)^{x}\left(e^{-\frac{\lambda}{q_{T}+1}}\right)^{N_{j}-x}
\end{aligned}
$$




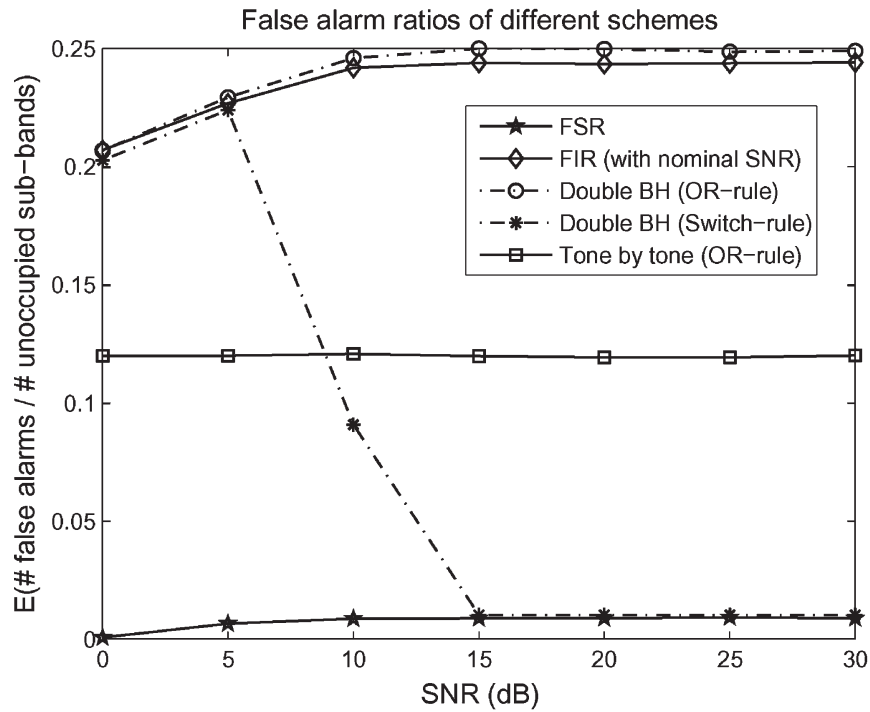

(a)

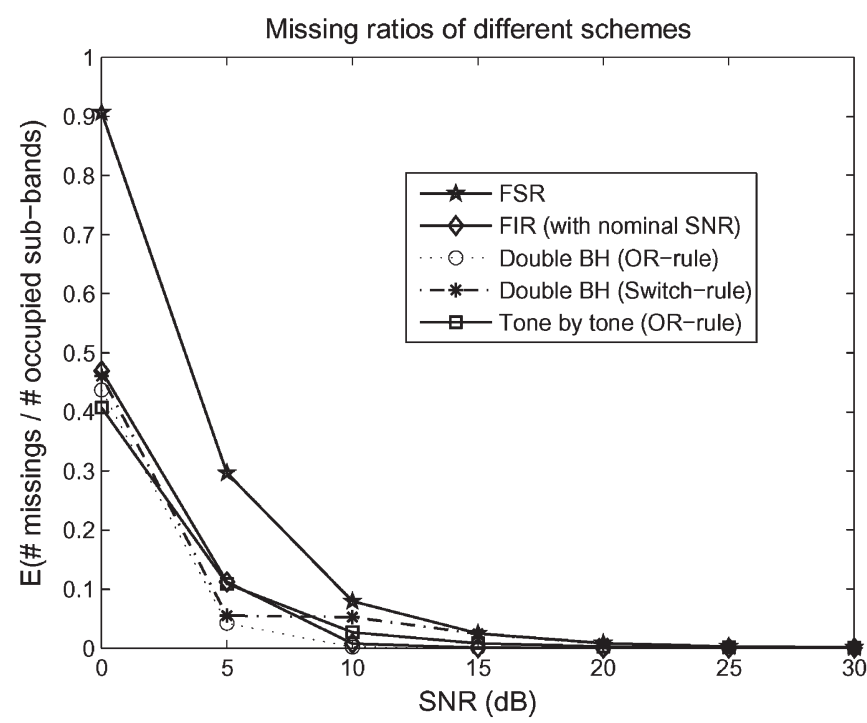

(b)

Fig. 5. FARs versus MRs with $M=100, K=20, S=30, \alpha_{T}=0.85$, $\alpha=0.05, \lambda_{T}=21, \lambda_{\mathrm{FSR}}=4$, and $\lambda_{\mathrm{FIR}}=1$. The nominal SNR for the FIR is set at $q^{*}=10 \mathrm{~dB}$, and the switching SNR is also set at $10 \mathrm{~dB}$ for the double $\mathrm{BH}$ procedures.

where $x_{j}$ is the number of observations out of $N_{j}$ that do not exceed $\lambda_{T}$. Then, apply the BH procedure for controlling the FIR at a significance level $\alpha_{T}$, as presented in Section III-B. The rejected subbands by the $\mathrm{BH}$ procedure are those with SNRs less than the threshold $\mathrm{SNR}_{T}$. The fusion center now applies the OR rule to the subbands rejected by the $\mathrm{BH}$ procedure and applies the AND rule to the rest of the subbands.

To implement the aforementioned SNR-switching rule for SS, the DF from the observers can be modified as follows. Suppose that the $\lambda_{T}$ of (12) is greater than the $\lambda$ of (8) such that $\lambda_{T}>\lambda>\lambda^{*}$. Then, the cognitive user reports 3 if $\left|Y_{i, j}\right|^{2}>\lambda_{T}$, reports 2 if $\lambda<\left|Y_{i, j}\right|^{2}<\lambda_{T}$, reports 1 if $\lambda^{*}<\left|Y_{i, j}\right|^{2}<\lambda$, and reports 0 otherwise. The total number of bits from each cognitive user is still two to apply the SNRswitching rule.
Simulation results for the SNR-switching rule are presented in Fig. 5 among other decision rules. For controlling the FIR, the nominal SNR $q^{*}$ is set at $10 \mathrm{~dB}$. The threshold SNR $q_{T}$ for switching between the OR and AND rules is also set at $10 \mathrm{~dB}$. In addition, the significance level is set at $\alpha_{T}=0.85$ for the $\mathrm{BH}$ procedure to identify the switching SNR. To evaluate the $p$-values, the thresholds for DF are set as $\lambda_{T}=21, \lambda=4$, and $\lambda^{*}=1$, respectively. Also shown in the figures for comparison is a rule denoted by "tone-by-tone" that takes the logic OR of all the DF of a subband to form the availability of the subband.

As can be seen from Fig. 5(a) and (b), respectively, the FAR of the SNR-switching rule is among the highest in the lowSNR regime, and the MR is comparable with that of the double $\mathrm{BH}$ and the tone-by-tone OR rules. Thus, the primary users are well protected from the MAI of cognitive access when their signals are already weak. However, in the high-SNR regime, both the FAR and the MR are well controlled at very low levels with the SNR-switching rule such that the system can enjoy throughput enhancement by allowing more aggressive access from the cognitive users, while, in the meantime, still preventing introducing MAI that is too excessive to the primary users.

\section{Classification of the Signal-to-Noise Ratio}

In Sections III and IV, the hypotheses for the BH procedure are only set for testing the existence of primary users. The SNR of each subband is not measured by the fusion center. If the SNR of each subband is also available to the fusion center, then the cognitive users may not only transmit through the idle subbands but may also be allowed to share the subbands with the primary users in a more generic cognitive manner [13], [14]. Motivated by the multilevel testing approach in [15], we extend the threshold-SNR-testing method in Section IV and study in this section a more precise SNR classification method based on multiple-hypothesis testing.

\section{A. SNR Classification with DF}

We partition the SNR into several levels and classify the SNR of each subband according to the predefined levels with the $\mathrm{BH}$ procedure. Considering the strength of practical wireless received signals, the received SNR is partitioned into a noise level plus $L_{\max }$ signal levels with the strength of each level equal to $5(L-1) \mathrm{dB}, L=1,2, \ldots, L_{\max }$.

To make use of the BH procedure for SNR classification, $L_{\max }+1$ null hypotheses and the corresponding probabilities against the hypotheses are set for the predefined SNR levels as follows.

1) The null hypothesis for the noise level

$H_{0}$ : The channel is idle with the presence of noise only

$$
P_{0}=\int_{\lambda_{0}}^{\infty} e^{-y} d y=e^{-\lambda_{0}} .
$$


2) The null hypothesis for the SNR level of $5(L-1) \mathrm{dB}$ $L=1,2, \ldots, L_{\max }$

$H_{L}$ : The channel is occupied with a signal strength at most $5(L-1) \mathrm{dB}$

$$
\begin{aligned}
& P_{L}=\int_{\lambda_{L}}^{\infty} \frac{1}{q_{L}+1} e^{-\frac{y}{q_{L}+1}} d y=e^{-\frac{\lambda_{L}}{q_{L}+1}} \\
& \text { with } q_{L}=10^{\frac{5(L-1)}{10}} .
\end{aligned}
$$

A cognitive user at a certain subband reports its observation according to the energy thresholds $\lambda_{L}$ defined for each hypothesis. There are a total of $L_{\max }+2$ observed levels being possibly received at the fusion center, which are $\left|Y_{i, j}\right|^{2}<\lambda_{0}$, $\lambda_{L-1} \leq\left|Y_{i, j}\right|^{2}<\lambda_{L}, L=1, \ldots, L_{\max }$, and $\left|Y_{i, j}\right|^{2} \geq \lambda_{L_{\max }}$. According to the discussion in Section III-C, the threshold $\lambda_{L}$ for each level can be determined according to

$$
-\left(q_{L}+1\right) \ln (\alpha) \leq \lambda_{L} \leq-\left(q_{L}+1\right) \ln \left(\frac{\alpha}{M}\right) .
$$

Given $P_{L}$, the corresponding $p$-values for each null hypothesis $H_{L}$ can be evaluated with

$$
p_{j, L}=\sum_{x=x_{j, L}}^{N_{j}}\left(\begin{array}{c}
N_{j} \\
x
\end{array}\right)\left(P_{L}\right)^{x}\left(1-P_{L}\right)^{N_{j}-x}
$$

where $x_{j, L}$ is the number of observations out of $N_{j}$ that exceeds $\lambda_{L}$. The BH procedure as stated in Section III-A can now be used to test each subband's SNR level. There are two types of methods for applying the $\mathrm{BH}$ procedure. For convenience of expression, they are referred to as the type-I and type-II testings in the sequel.

1) Type-I testing: In this approach, the $\mathrm{BH}$ procedure is first applied to test the $M$ subbands with respect to a single SNR level each time. The $p$-values, which are sorted in each $\mathrm{BH}$ procedure, come from different subbands. The results after each testing only indicate whether the subbands exceed the SNR level under test. After collecting the results tested against each SNR level, the fusion center then decides the SNR region for each subband according to the rule stated in (19).

2) Type-II testing: This approach does not test the $M$ subbands for one SNR level at a time. Instead, it applies the $\mathrm{BH}$ procedure to test the $L_{\max }+1$ hypotheses of SNR for a single subband each time. As such, the threshold for each hypothesis is set at the middle of the range

$$
\begin{aligned}
-\left(q_{L}+1\right) \ln (\alpha) & \leq \lambda_{L} \\
& \leq-\left(q_{L}+1\right) \ln \left(\frac{\alpha}{L_{\max }+1}\right) .
\end{aligned}
$$

The $p$-values used for the $\mathrm{BH}$ procedure in each subband are calculated with the decision bits reporting according to the different thresholds. With the $p$-values of the subband, the corresponding SNR region is determined again with the rule in (19), shown at the bottom of the page, where $\bar{q}_{j}, j=1, \ldots, M$, is the classified SNR region for subband $j$.

\section{B. SNR Classification with EF}

Similar to the methods just presented in Section V-A for SNR classification with DF, EF can also be used to classify the received SNR strength of each subband. Different from the $p$-values in (17) that requires $P_{L}$ for decision fusion, the $p$-values for energy fusion only depend on the total observed energy $\sum_{i=1}^{N_{j}}\left|Y_{i, j}\right|^{2}$ in each subband and can easily be calculated with the CCDF of the Gamma distribution. The hypotheses and the corresponding $p$-values are listed below.

1) The null hypothesis for the noise level

$H_{0}$ : The channel is idle with the presence of noise

only

$$
\begin{aligned}
p_{j, 0}= & \operatorname{Pr}\left\{Y \geq \sum_{i=1}^{N_{j}}\left|Y_{i, j}\right|^{2} \mid H_{0}\right\} \\
= & \int_{\sum\left|Y_{i, j}\right|^{2}}^{\infty} \frac{y^{\left(N_{j}-1\right)} e^{-y}}{\Gamma\left(N_{j}\right)} d y, \quad j=1, \ldots, M .
\end{aligned}
$$

2) The null hypothesis for the SNR level of $5(L-1) \mathrm{dB}$, $L=1,2, \ldots, L_{\max }$

$H_{L}$ : The channel is occupied with a signal strength at most $L \mathrm{~dB}$

$$
\begin{array}{r}
p_{j, L}=\operatorname{Pr}\left\{Y \geq \sum_{i=1}^{N_{j}}\left|Y_{i, j}\right|^{2} \mid H_{L}, q_{L}\right\} \\
=\int_{\sum\left|Y_{i, j}\right|^{2}}^{\infty} \frac{y^{\left(N_{j}-1\right)} e^{-\frac{y}{q_{L}+1}}}{\Gamma\left(N_{j}\right)\left(q_{L}+1\right)^{N_{j}}} d y \\
\text { with } \quad q_{L}=10^{\frac{5(L-1)}{10}}, \quad j=1, \ldots, M .
\end{array}
$$

$$
\bar{q}_{j}=\left\{\begin{array}{cc}
\text { only noise, } & \text { if no } \mathrm{H} \text { hypothesis is rejected } \\
\text { noise } \sim 0 \mathrm{~dB}, & \text { if only } H_{o} \text { is rejected } \\
5(L-1) \sim 5 L \mathrm{~dB}, & \text { if } H_{L} \text { is the maximum of rejected hypothesis }
\end{array}\right\}
$$


The type-I and type-II methods for applying the $\mathrm{BH}$ procedure in Section V-A can also be used here to test the SNR region with EF. The final decision rule still follows (19).

\section{Simulation Results}

Some numerical experiments are conducted to evaluate the performance of the proposed decision fusion methods. Suppose that there are $M=100$ subbands going to be sensed by $K=20$ cognitive users. Each subband may be occupied by a primary user with equal probability, and each cognitive user randomly selects $S$ consecutive subbands to measure the energies and reports the corresponding sensing results to the fusion center. The simulation results are summarized and discussed in two parts. The first part presents the performance of the proposed scheme in SS, and the second part discusses its effectiveness in SNR classification.

\section{A. Performance in SS}

In Section III, a rather idealized assumption that occupied subbands have the same received SNR is made to verify the algorithms' performance in different SNRs. To evaluate the effectiveness of the algorithms in practical communication environments, we consider three kinds of simulation settings in this section to account for the possible fading and shadowing effects in wireless channels. For the BH procedure using decision fusion, the energy threshold is set to $\lambda=4$ to control the FSR, and it is set to $\lambda=1$ to control the FIR. In addition, the $\mathrm{BH}$ procedure is conducted at a significance level of $\alpha=0.05$.

1) Subband-Dependent Setting: In this simulation setting, cognitive users experience the same SNR in a subband, while we may see different SNRs in different subbands. The SNR of each occupied subband is randomly chosen from 0 to $30 \mathrm{~dB}$.

Since the SNR is the same for all users in each subband, the FSR and FIR approaches introduced in Section III can directly be applied here to the test of the existence of primary users. In addition, to assess the feasibility of controlling the FIR under this channel condition, ideal SNRs are used to evaluate the $p$-values that are already defined in (5) and (7) for the FSR and FIR, respectively.

2) User-Dependent Setting: In this case, each cognitive user has a uniform SNR in all the subbands under his/her test, while different users experience different SNRs. This simulation setting can reasonably be applied to study the performance in a less frequency-selective channel in which the consecutive subbands scanned by a cognitive user have similar SNRs.

Under this channel assumption, the decision bits obtained from different users in a single subband may reference different levels of SNRs. This will make it infeasible to use ideal SNRs in assessing the FIR. Nevertheless, it is still valid to evaluate the FSR and the FIR operating on a nominal SNR. The corresponding $p$-values are also defined in Section III.

3) General Setting: The most general and practical communication environment is considered in this setting, where each cognitive user experiences different SNRs in different

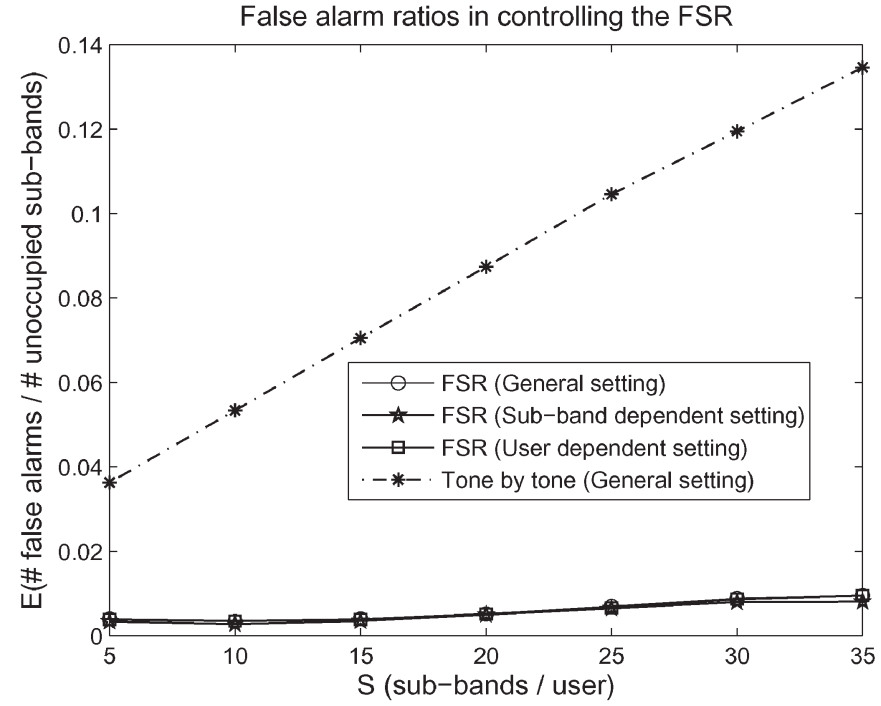

(a)

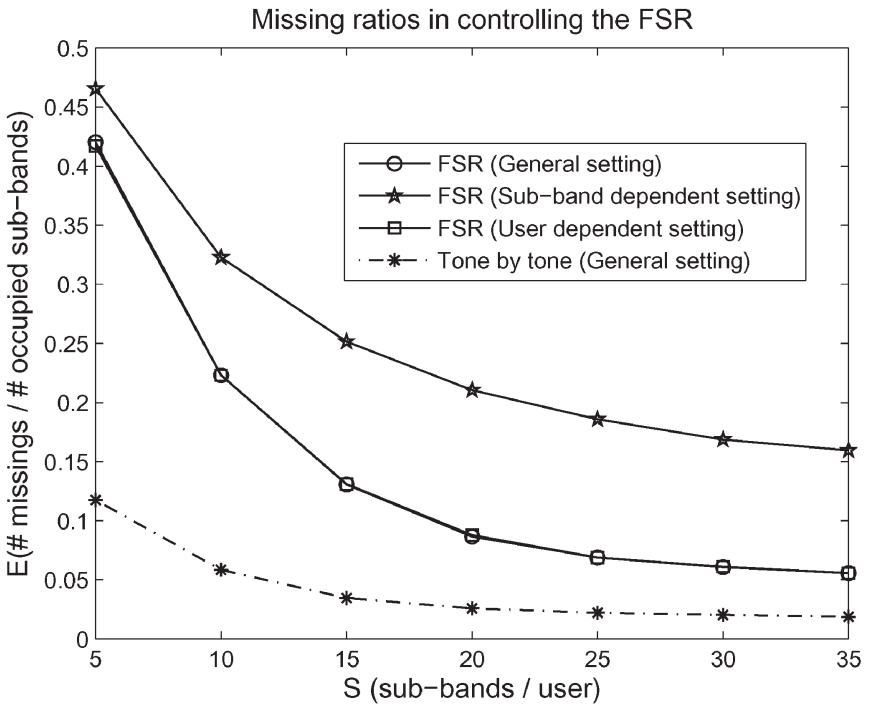

(b)

Fig. 6. FARs versus MRs of controlling the FSR in different channel settings with $M=100, K=20, \lambda_{\mathrm{FSR}}=4$, and $\alpha=0.05$.

subbands. Similar to the user-dependent setting, ideal SNRs are not provided either in evaluating the FIR in this case. Other than this, the $p$-values are similar to the previous settings.

The performance of the proposed cooperative sensing methods are simulated and compared in Figs. 6-8. For comparison, the commonly used tone-by-tone OR rule, which tests one subband at a time and declares the channel occupied if more than one decision bits are one, is also studied in simulations. In controlling the FSR, the FARs remain around 0.01 in Fig. 6(a) for all simulation settings and the MRs decrease with the increasing number of scanning subbands (S) per user. On the other hand, to control the FIR, the MRs can also be suppressed at fairly low levels, as shown in Fig. 7(b) for the user-dependent and general settings. The behavior that the MRs of the subband-dependent setting are higher than the other two settings in Figs. 6(b) and 7(b) is mainly due to the fact that the SNR is fixed for each subband. Therefore, a large portion of the observations is done on low-SNR signals, hence degrading the sensing performance. 


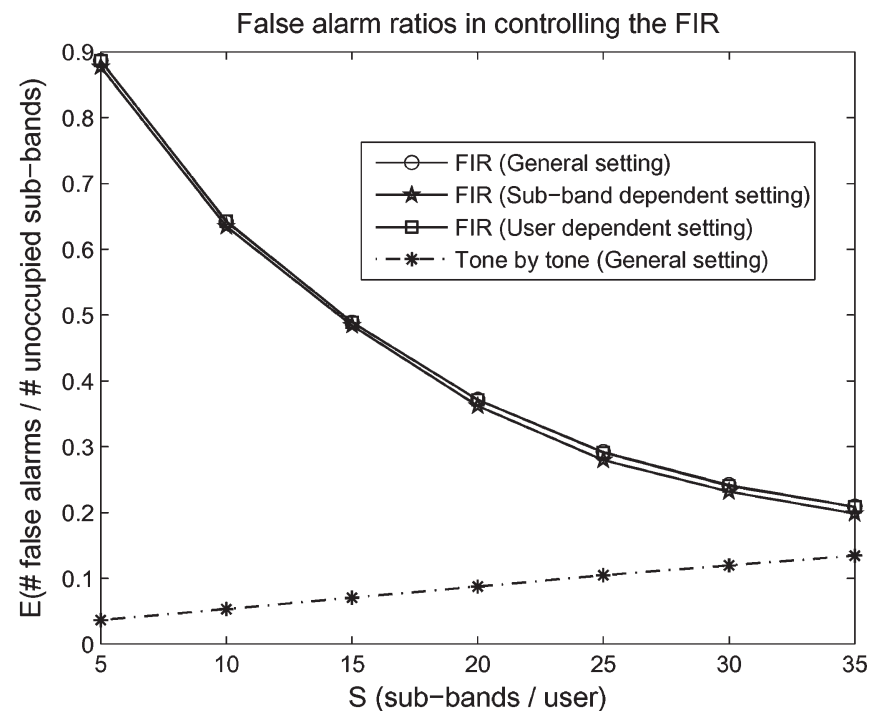

(a)

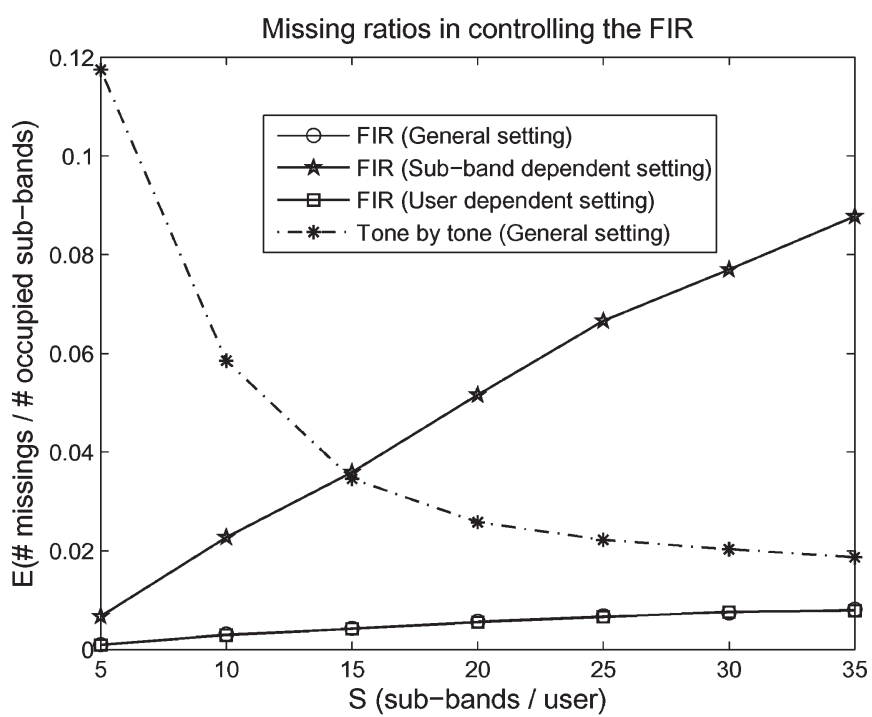

(b)

Fig. 7. FARs versus MRs of controlling the FIR in different channel settings with $M=100, K=20, \lambda_{\mathrm{FIR}}=1$, and $\alpha=0.05$. The nominal SNR is set at $q^{*}=10 \mathrm{~dB}$.

Judging from these results, we see that the number of scanning subbands for each user should be around 25-35 to control the FIR to obtain a balanced FAR and MR.

The simulation results for the double $\mathrm{BH}$ procedures are presented in Fig. 8. It is shown in Fig. 8(a) that the SNR-switching rule can drastically reduce the FAR, even if the number of scanning subbands per user is very small. On the other hand, it still maintains an acceptable MR, as shown in Fig. 8(b).

\section{B. Performance of SNR Classification}

In the simulation studies for SNR classification, the subbanddependent setting is adopted to simulate the channel condition in which each occupied subband has an individual and fixed SNR randomly chosen from 0 to $30 \mathrm{~dB}$. The entire simulated range of the SNR is partitioned into six nonoverlapping regions, each of them being $5 \mathrm{~dB}$ in width. The SNR of each subband is

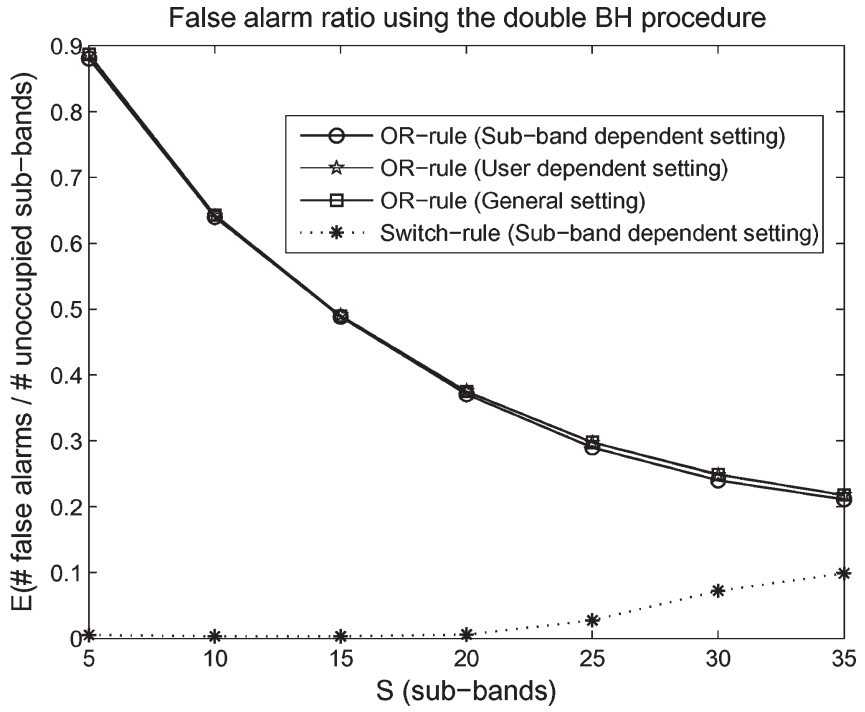

(a)

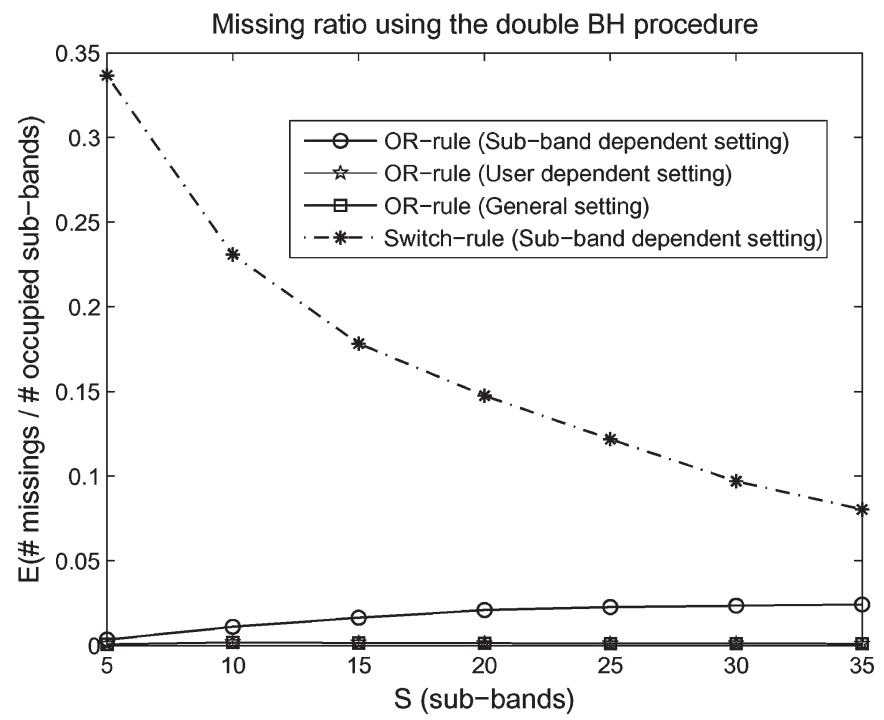

(b)

Fig. 8. FARs versus MRs of using the double $\mathrm{BH}$ procedure in different simulation settings with $M=100, K=20, \lambda_{T}=21, \lambda_{\mathrm{FSR}}=4, \lambda_{\mathrm{FIR}}=$ $1, \alpha_{T}=0.85$, and $\alpha=0.05$. The nominal SNR for the FIR is set at $q^{*}=$ $10 \mathrm{~dB}$, and the switching SNR is also set at $10 \mathrm{~dB}$ for the double $\mathrm{BH}$ procedures.

classified according to the predefined SNR levels at the fusion center with the rule in (19). The results of classification are shown in pie charts in Figs. 9 and 10. The results of type-I testing are demonstrated in Fig. 9, and the results of type-II testing are shown in Fig. 10. The slices marked with "correct" are the percentage of correct estimation. The slices marked with "-1 level" mean that the estimated SNRs are one level lower than the true SNRs. Similarly, the slices marked with " +1 level" show the percentage of results that are one level higher than the true SNRs.

By comparing the pie charts in Figs. 9 and 10, it is shown that type-II testing gives better results than type I, and methods with EF outperform their counterparts that use DF. The reason is that the $\mathrm{BH}$ procedure of type I tests one hypothesis each time for all subbands whose observations may come from sources of different SNRs. On the other hand, type II performs multiplehypothesis testing for one subband each time, based on the 


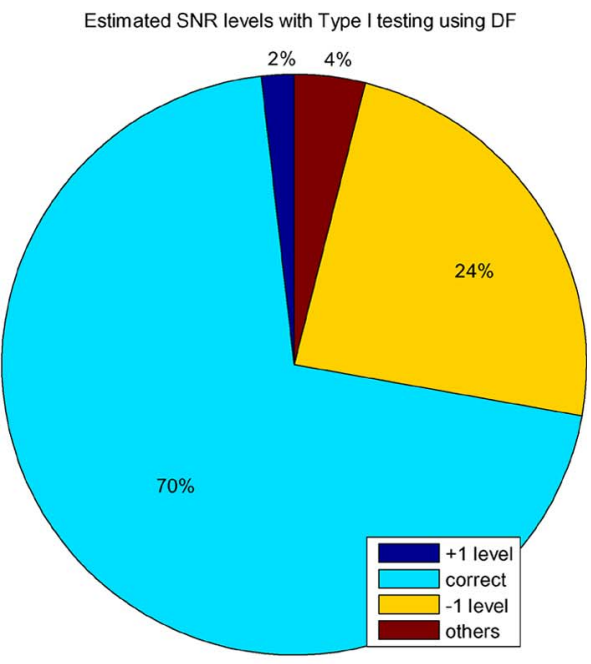

(a)

Estimated SNR levels with Type I testing using EF

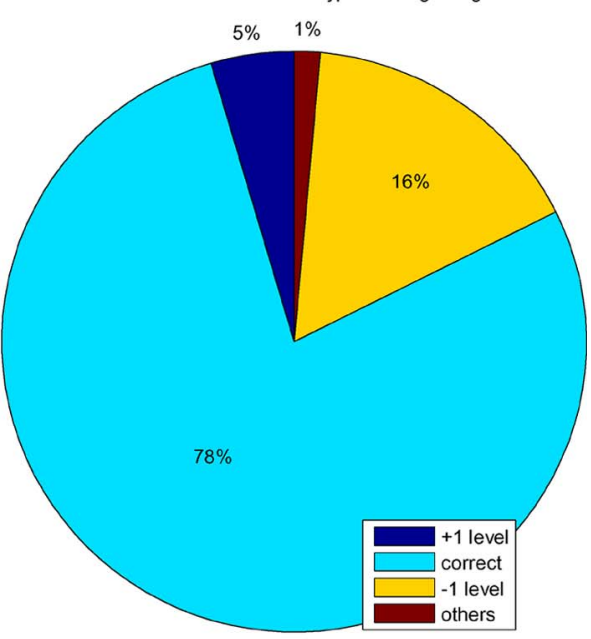

(b)

Fig. 9. Results of SNR classification using type-I testing with $M=100$, $K=20, S=30$, and $\alpha=0.85$. (a) Results with DF. (b) Results with EF.

observations on signal sources that are generated with the same SNR. Consequently, the $p$-values for type-II testing in each subband are calculated with the same CCDF (17) of different $P_{L}$ that correspond to different hypotheses. On the other hand, for type-I testing, the $p$-values for each $\mathrm{BH}$ procedure are calculated with the CCDFs of different $N_{j}$ and $P_{L}$ of different subbands, causing the variation in testing results to be a little higher.

In addition to the performance differences between type-I and type-II testings, the biases of the estimates with DF are more pronounced than the results with EF. This is due to the fact that the $\mathrm{BH}$ procedure done on discrete statistics tends to control the FDR at a level even lower than the BH with EF does [12], thus acting more conservatively in rejecting the null hypotheses, which, in turn, results in lower estimates of SNRs than the true levels. Fig. 9(a) shows that the percentage of results that are one level lower is $24 \%$, while the percentage of the other error estimates is $6 \%$ in total. In comparison, the error of one-level-lower results drops by $8 \%$ in Fig. 9(b) with EF. On the other hand, for type-II testing, the percentage of onelevel-lower results reduces to $14 \%$ in Fig. 10(a), which is only

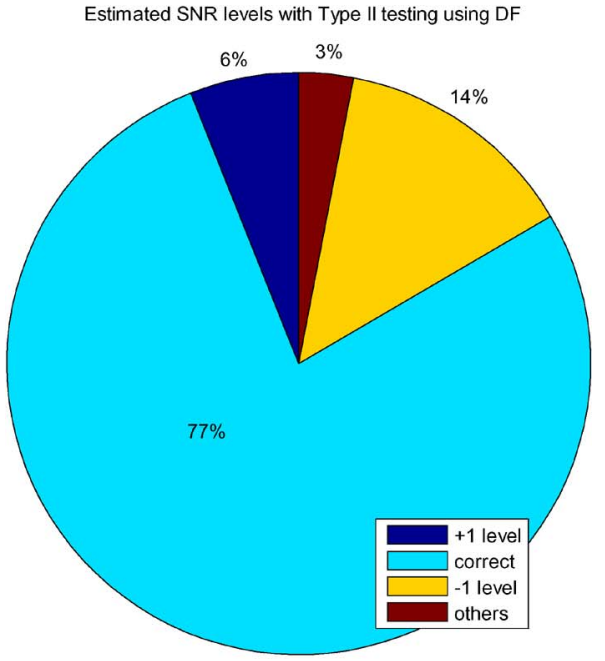

(a)

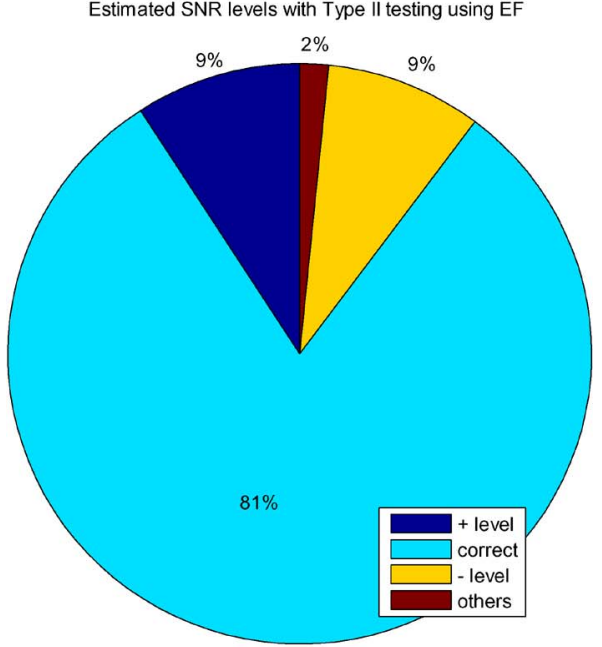

(b)

Fig. 10. Results of SNR classification using type-II testing with $M=100$, $K=20, S=30$, and $\alpha=0.85$. (a) Results with DF. (b) Results with EF.

$8 \%$ greater than the percentage of one-level-higher results. In contrast, there is no bias in Fig. 10(b) with EF.

\section{CONCLUSION}

The decision fusion method has been presented for cooperative SS of wideband CR systems using multiple-hypothesis testing. Both the FSR and FIR derived from the notion of FDR have been shown to be controlled under the desired levels by the $\mathrm{BH}$ procedure. Simulations have shown that the decision fusion method can achieve performance comparable with the energyfusion method while saving the overhead to report continuous values. Moreover, the double $\mathrm{BH}$ procedure in conjunction with the SNR-switching rule has achieved the lowest FAR and MR at a high SNR while still enjoying a low MR and a relatively high FAR in the low-SNR regime. This can potentially improve the system throughput by allowing for more aggressive cognitive access in the high-SNR regime, while preventing MAI that is too excessive to the primary users in the low-SNR regime.

On the other hand, for SNR classification, simulations have also shown that the SNRs can be classified within one level plus 
or minus the true SNR strength with more than a $96 \%$ chance. This allows the system to employ more generic protocols such as the concurrent transmissions of the primary and secondary users' signals for cognitive access.

\section{REFERENCES}

[1] M. Sherman, A. N. Mody, R. Martinez, C. Rodriguez, and R. Reddy, "IEEE standards supporting cognitive radio and networks, dynamic spectrum access, and coexistence," IEEE Commun. Mag., vol. 46, no. 7, pp. 72-79, Jul. 2008.

[2] A. Ghasemi and E. S. Sousa, "Collaborative spectrum sensing for opportunistic access in fading environments," in Proc. 1st IEEE Symp. New Frontiers Dyn. Spectrum Access Netw., Baltimore, MD, Nov. 2005, pp. 131-136.

[3] C. Sun, W. Zhang, and K. B. Letaief, "Cluster-based cooperative spectrum sensing in cognitive radio systems," in Proc. IEEE ICC, Glasgow, U.K., Aug. 2007, pp. 2511-2515.

[4] C. Sun, W. Zhang, and K. B. Letaief, "Cooperative spectrum sensing for cognitive radios under bandwidth constraints," in Proc. IEEE WCNC, Hong Kong, Mar. 2007, pp. 1-5.

[5] G. Atia, E. Ermis, and V. Saligrama, "Robust energy efficient cooperative spectrum sensing in cognitive radios," in Proc. IEEE SSP, Madison, WI, Aug. 2007, pp. 502-506.

[6] Y. Benjamini and Y. Hochberg, "Controlling the false discovery rate: A practical and powerful approach to multiple testing," J. R. Stat. Soc. Ser. $B$, vol. 57, no. 1, pp. 289-300, 1995.

[7] S. Holm, "A simple sequentially rejective multiple test procedure," Scand. J. Stat., vol. 6, no. 2, pp. 65-70, 1979.

[8] R. J. Simes, "An improved Bonferroni procedure for multiple tests of significance," Biometrika, vol. 73, no. 3, pp. 751-754, 1986.

[9] G. Hommel, "A stagewise rejective multiple test procedure based on a modified Bonferroni test," Biometrika, vol. 75, no. 2, pp. 383-386, 1988.

[10] Y. Hochberg, "A sharper Bonferroni procedure for multiple tests of significance," Biometrika, vol. 75, no. 4, pp. 800-803, 1988.

[11] D. M. Rom, "A sequentially rejective test procedure based on a modified Bonferroni inequality," Biometrika, vol. 77, no. 3, pp. 663-665, 1990.

[12] Y. Benjamini and D. Yekutieli, "The control of the false discovery rate in multiple testing under dependency," Ann. Stat., vol. 29, no. 4, pp. 11651188, Aug. 2001.

[13] N. Devroye, P. Mitran, and V. Tarokh, "Achievable rates in cognitive radio channels," IEEE Trans. Inf. Theory, vol. 52, no. 5, pp. 1813-1827, May 2006

[14] A. Tajer, N. Prasad, and X. Wang, "Distributed beamforming and rate allocation in multi-antenna cognitive radio networks," in Proc. IEEE ICC, Dresden, Germany, Jun. 2009, pp. 1-6.

[15] P.-J. Chung, J. F. Bohme, C. F. Mecklenbrauker, and A. O. Hero, "Detection of the number of signals using the Benjamini-Hochberg procedure," IEEE Trans. Signal Process., vol. 55, no. 6, pp. 2497-2508, Jul. 2007.

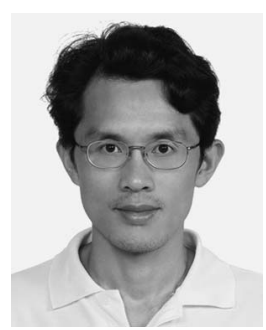

Sau-Hsuan Wu (S'03-M'04) received the B.S. and M.S. degrees in engineering science from the National Cheng Kung University, Tainan, Taiwan, in 1990 and 1993, respectively, and the Ph.D. degree in electrical engineering from the University of Southern California, Los Angeles, in 2003.

From 1993 to 1995, he served in the Army of Taiwan. From 1995 to 1999 , he was a Circuit and System Design Engineer in Taiwan. From 2003 to 2004, he was a Postdoctoral Research Fellow with the Department of Electrical Engineering, University of Southern California. From 2004 to 2005, he was a Technical Consultant for Winbond Electronics Corporation America, developing wireless multipleinput-multiple-output orthogonal frequency-division multiplexing products. Since 2005, he has been an Assistant Professor with the Department of Communication Engineering, National Chiao Tung University, Hsinchu, Taiwan. His research interest is in the application of signal processing theory to wireless communication systems.

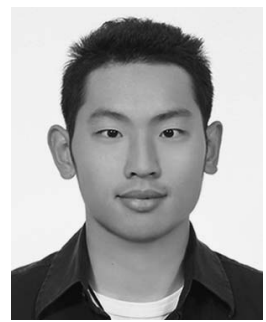

Chao-Yuan Yang received the B.S. degree in electrical engineering from the National Chung Hsing University, Taichung, Taiwan, in 2006 and the M.S degree in communication engineering from the $\mathrm{Na}$ tional Chiao Tung University (NCTU), Hsinchu, Taiwan, in 2008. He was a graduate student with the Mobile Broadband Wireless Communication Laboratory, NCTU, from September 2006 to September 2008.

He is currently with the Department of Communication Engineering, NCTU. His research interests include multiple-input-multiple-output systems, Third-Generation Partnership Project spatial channel modeling, cooperative communication, and cognitive radio.

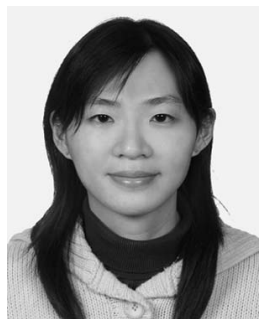

D.-H. Tina Huang received the B.S. and M.S. degrees in engineering and system science from the National Tsing Hua University, Hsinchu, Taiwan, in 2002 and 2005, respectively. She is currently working toward the Ph.D. degree in communication engineering with the Department of Communication Engineering, National Chiao Tung University, Hsinchu.

From May 2007 to March 2008, she was a Contract-Based Engineer with MediaTek Inc. and joined the meeting of the IEEE $802.16 \mathrm{~m}$ Broadband Wireless Access Standards. Her research interests are in the areas of machine learning, channel coding, signal processing theory, and their applications in the wireless communication system and in biomedical engineering. 